\title{
ANÁLISIS COMPARATIVO DE LA ESTRUCTURA Y BIODIVERSIDAD DE UN SABINAR LITORAL Y SU SUCESIÓN EN EL ESPACIO NATURAL DOÑANA
}

\author{
R. Cámara Artigas y J.R. Alonso García \\ Departamento de Geografía Física y Análisis Geográfico Regional \\ Universidad de Sevilla, C/ Doña María Padilla, s/n, 41004 Sevilla \\ racamara@us.es \\ jralonso002@hotmail.com
}

\begin{abstract}
Resumen: Se analiza y compara la estructura, composición y biodiversidad de un sabinar litoral y su matorral de sucesión en el Médano del Asperillo (Espacio Natural Doñana, al suroeste de España) a través de diagramas de estructura, el índice de valor de importancia e índices de biodiversidad alfa, paramétricos y no paramétricos. Dicho análisis permite establecer el ritmo diacrónico, lo cambios en la estructura y composición, así como en la biodiversidad entre las dos comunidades vegetales: el matorral de monte blanco desarrollado sobre un área desforestada desde el año 1947 hasta la actualidad, y una fragmento de sabinar en el sitio de las Atarazanas desde esa misma fecha.
\end{abstract}

Palabras clave: sabinar, Doñana, monte blanco, biodiversidad alfa.

\begin{abstract}
The purpose of this work, consisted in the analysis and comparison of the structure, composition and biodiversity of the Coastal Phoenicean juniper and its Scrub Succession, throughout structure diagrams, importance value and both parametrical/no parametrical biodiversity alpha indexes. These species can be found in the Natural Space of Doñana, located at the southwest of Spain, specifically at Medano del Asperillo. This analysis, allows to establish the diachronically rhythm, structure, composition and biodiversity changes between both plant communities: The scrub monte bllanco, based upon the deforested area since 1947 and a sample of the Sabinar taken from las Artaranzas of the same year.
\end{abstract}

Keywords: Phoenicean juniper, Doñana, monte blanco, alpha biodiversity.

Recibido: 13-04-2015. Aceptado: 25-06-2015. 


\section{Introducción}

El sabinar litoral es una formación arbórea y arbustiva monoespecífica, con dominancia de Juniperus phoenicea subsp. turbinata (Guss.) Nyman., que es, junto a los pinares, el monte blanco y el monte negro, característica en la superficie de arenas que conforma el Espacio Natural Doñana.

El interés en estudiar esta comunidad vegetal se basa en que constituye una entidad propia, diferenciada del resto de comunidades, que se distribuye formando un bosque en un sector muy localizado del Espacio Natural Doñana. A pesar de ser una comunidad autóctona, más que gran parte de la superficie de pinares de éste área protegida, es poco conocida en su composición, estructura, dinámica y biodiversidad.

La composición específica del sabinar ha sido estudiada desde la década de los 80. El primer trabajo que se realizó con el método fitosociológico fue el estudio sistemático de Rivas-Martínez et al. (1980) en el que se inventarió una superficie $260 \mathrm{~m}^{2}$, en 12 parcelas. De ellas, la mayoría se levantaron en Marismillas, al sur del Parque Nacional, junto a la desembocadura del Guadalquivir. Sólo una, con $20 \mathrm{~m}^{2}$ de superficie, se realizó en la Reserva Biológica, y ninguna en el Médano del Asperillo. Bejarano (2004) realizó un ensayo tipológico de los sabinares costeros de Andalucía occidental en función de los criterios de composición florística, abundancia-dominancia por especie y riqueza florística. De Las Heras (2007) y López (2009) en sus tesis doctorales abordan también desde el método fitosociológico el estudio de la vegetación del Manto Eólico de Doñana, extendiendo su estudio al Médano del Asperillo. Por su parte Cámara y Bejarano (2010) realizaron una comparación metodológica, entre el método fitosociológico y el desarrollado en éste artículo, de las parcelas de sabinar situadas al norte del Charco del Toro, confrontando los resultados obtenidos. El último estudio sistemático con el método fitosociológico realizado en el sabinar de la Reserva Biológica fue el de R. Bejarano, A. Romo y M. Salvá (Bejarano et al. 2014) en el que se realizaron 23 inventarios $\left(460 \mathrm{~m}^{2}\right)$ y en el que se aportó, entre otros resultados, que el $73 \%$ de los taxones inventariados era herbáceas.

En los últimos años, a través de nuestro Grupo de Investigación, se han realizado nuevos trabajos de investigación, con el método aquí desarrollado, en los sabinares de la Reserva Biológica de Doñana (Cámara, 2013; Cámara, Gómez et al. 2014; Cámara, Díaz del Olmo, et al. 2014), concretamente en El Ojillo, Sabinar del Marqués y Charco del Toro, inventariándose una superficie de $4.600 \mathrm{~m}^{2}$ en 46 transectos, que han aportado un mayor conocimiento de la estructura, composición y biodiversidad de esta comunidad vegetal.

Finalmente, se han realizado, también recientemente, trabajos sobre el comportamiento fenológico del sabinar como respuesta a eventos extremos de sequía (Díaz-Delgado, 2014). 
Desconocemos cual es el ritmo de remontada biológica y los cambios en la biodiversidad de los sabinares post intervención antrópica. La hipótesis de partida es que las situaciones de morfogénesis e inestabilidad han originado las morfologías eólicas de dunas, y para que estas se desarrollen ha sido preciso la ausencia de una cobertura vegetal densa. De forma contraria se asume que el desarrollo de dicha cobertura vegetal ha favorecido la estabilidad del sistema y el desarrollo de formaciones vegetales ecológicamente más maduras sobre las dunas estabilizadas por las propias formaciones vegetales. Las fases de desestabilización, ya sean por cambio climático o por acción antrópica, han favorecido, en consecuencia, la reactivación de las formaciones eólicas. Pero no sabemos en cuanto tiempo tiene lugar en ésta sucesión y cuáles son los cambios estructurales y en su riqueza y abundancia.

El objetivo de este trabajo es establecer cuáles son los cambios cualitativos en la estructura y composición de las formaciones vegetales que se comparan, sabinar y matorral de sustitución sobre arenas, y los cambios cuantificables mediante el cálculo de índices de biodiversidad paramétricos y no paramétricos, para así establecer el ritmo de la sucesión y los cambios asociados a ésta, y el estado de perturbación ambiental en que se encuentran las comunidades vegetales estudiadas.

Para ello hemos estudiado el avance de la vegetación con un matorral de cistáceas en un sector que aparecía desprovisto de vegetación en Cuesta Maneli en 1947, y lo hemos comparado con la vegetación de sabinar persistente desde esa misma fecha en Las Atarazanas, que está localizado en la cubeta de una duna parabólica.

\section{2. Área de estudio}

El sitio de Las Atarazanas se encuentra ubicado en el médano del Asperillo, que se localiza en la costa entre Mazagón y Matalascañas en el Espacio Natural Doñana, al suroeste de Andalucía. La costa se configura como un acantilado estabilizado con 60 a $100 \mathrm{~m}$. de desnivel, que es el resultado de una paleolínea costera (Borja y Díaz del Olmo, 1994a) modelada sobre el Manto Eólico litoral (MEL) de Doñana (figura 1).

Los sabinares de Juniperus phoenicea subsp. turbinata (Guss.) Nyman geobotánicamente colonizan los arenales y sistemas de dunas fitoestabilizadas del Alto Manto Eólico Seco (AMEs), que junto al Alto Manto Eólico Húmedo (AMEh) y el Manto Eólico de Dunas Semiestables (MEDS) constituyen el mencionado Manto Eólico Litoral de El Abalario-Doñana (Borja y Díaz del Olmo, 1994b; Montes, 1998; Zazo et al., 1999). Los sabinares sólo se encuentran formando bosque en el AMEs y ocasionalmente en el Médano del Asperillo, que se sitúa entre Mazagón al Norte y Matalascañas al Sur. Fuera de estos ámbitos en el Espacio Natural Doñana, que engloba el Asperillo y el propio Parque Nacional, los sabinos se encuentran dispersos y entremezclados con el pinar. Al sur del Parque Nacional, en Marismillas, forman un bosque mixto con el pinar. 


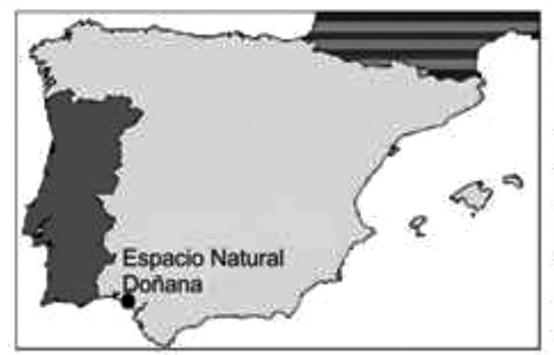

Contacto Ecodistritos Marisma/Eolico-Costero

Bajo Manto Eólico de Dunas Fitoestables Alto Manto Eólico Húmedo de Dunas Fitoestable: Alto Manto Eólico Seco de Dunas Fitoestables Manto Eolico de Dunas Semiestables Manto Eollico de Dunas Activas Antropizado

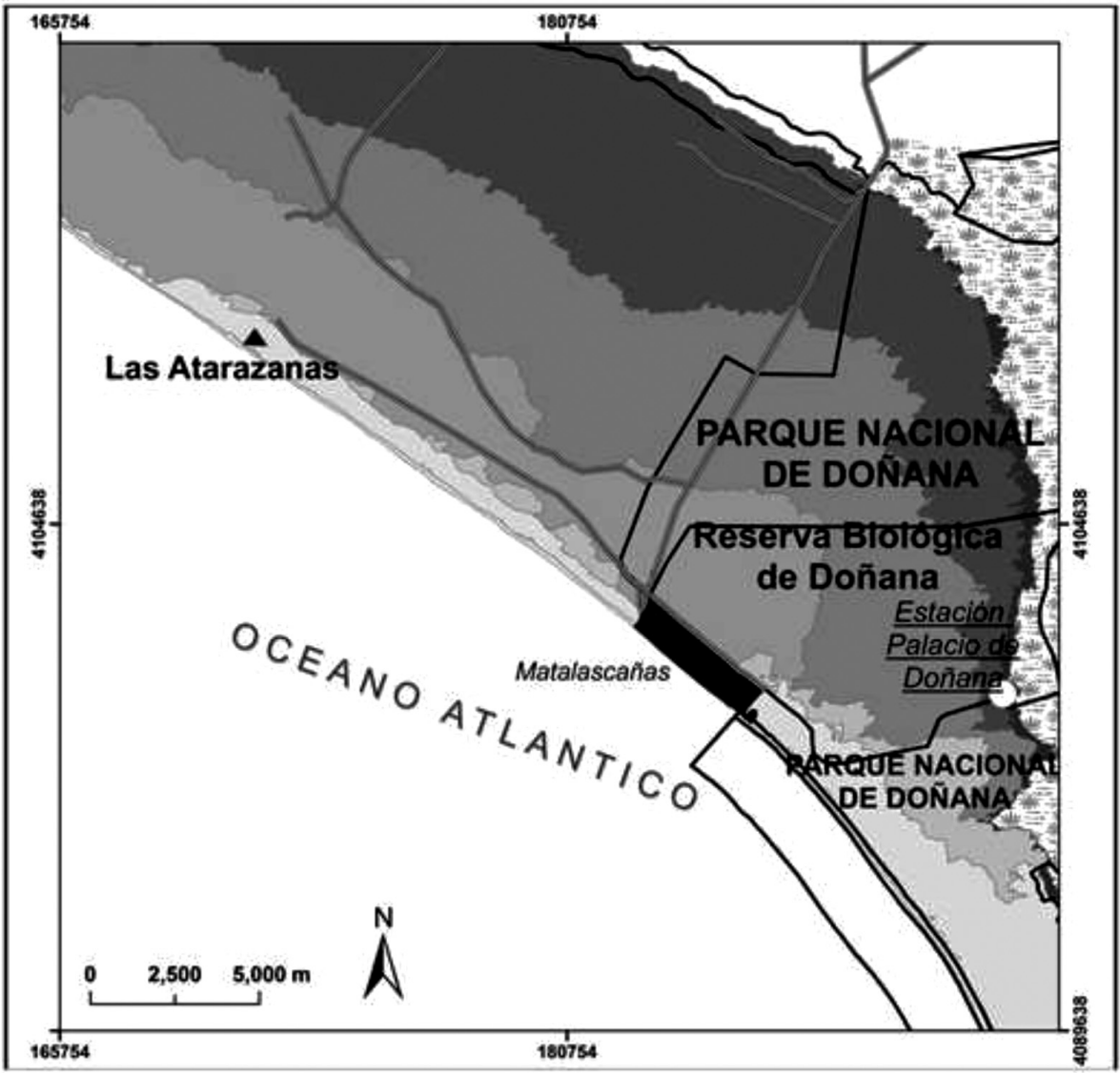

Figura 1. Ubicación de Las Atarazanas en el Espacio Natural Doñana. Fuente: Elaboración propia. 
La observación de las fotos áreas de 1947 de la Fototeca del Instituto Geográfico Nacional (http://fototeca.cnig.es/) (figura 2) en comparación con el fotograma aéreo de 2009 (vuelo fotogramétrico 2009 Junta de Andalucía) nos han permitido identificar en el sitio de Las Atarazanas un resto de vegetación que permaneció sin ser alterado desde al menos finales de los años 40. Mientras, todo el entorno aparece desprovisto de vegetación por causas desconocidas, que atribuimos a la acción antrópica por su sistematización y extensión, dejando reductos muy localizados.

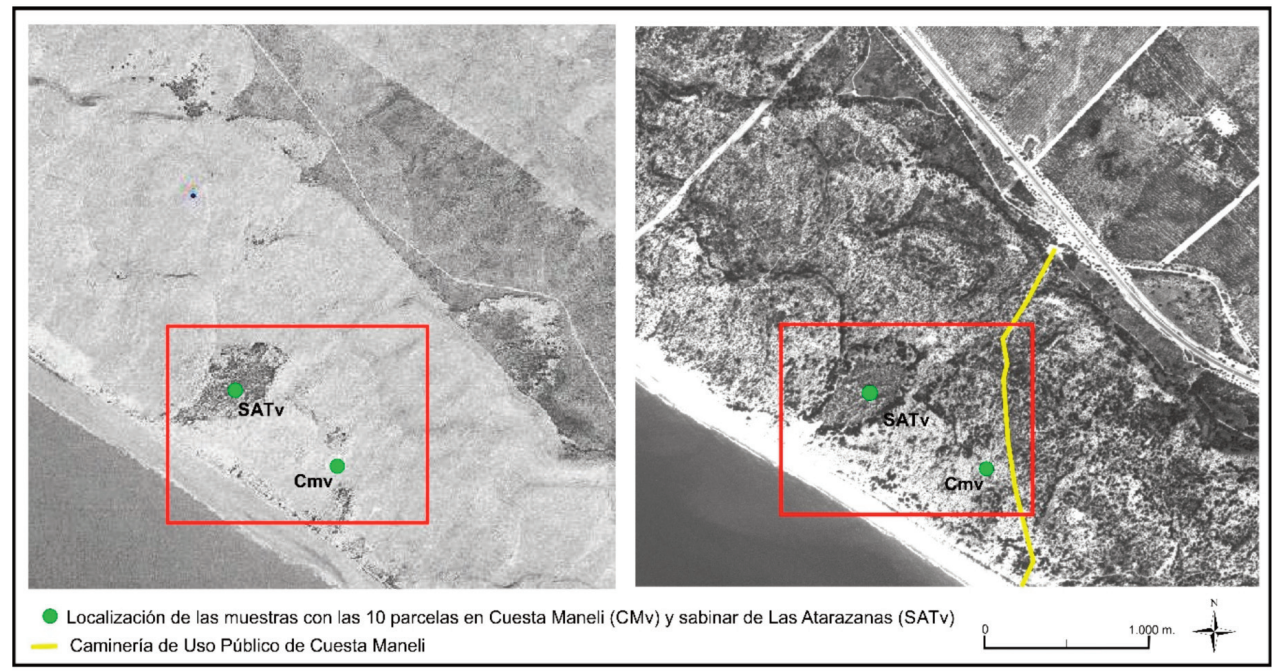

Figura 2. Comparación entre los fotogramas aéreos de 1947 y 2009 con la ubicación de las muestras de inventario. Elaboración propia a partir de fotogramas aéreos de 1947 del IGN y de 2009 de la Junta de Andalucía.

El propio toponímico de las Atarzanas hace referencia al uso del sabinar. El manejo de la madera del sabino ha sido recogido para el conjunto de Doñana por García Novo et al. (2007, pp. 119 y 120), que habla de la deforestación del sabinar de este entorno a finales del siglo XVIII para la almadraba de Torre Carbonera, al sur de Doñana, la instalación de los viñedos, también en el extremo sur de Doñana, y el sobrepastoreo.

\section{Métodos y técnicas}

Para el estudio de las formaciones vegetales se han realizado 22 parcelas de $100 \mathrm{~m}^{2}$. Para ello se ha utilizado un método de inventario y análisis de los atributos vegetales basado en el muestreo de las especies leñosas presentes denominado MIFC (Cámara 
y Díaz del Olmo, 2013) que permite identificar los individuos en términos absolutos y relativos, y los siguientes atributos: frecuencia, densidad, biotipos, estructura vertical, estructura horizontal (cobertura), y diámetro a la altura del pecho (DAP). A partir de ellos se puede:

- Calcular el Índice de Valor de Importancia (IVI) que es la suma de la abundancia, frecuencia y dominancia o área basal expresadas en valores relativos (Krebs 1985).

- Aplicar índices de diversidad alfa paramétricos y no paramétricos para establecer el valor de biodiversidad de las diferentes formaciones.

El MIFC tiene la virtualidad de identificar las formaciones vegetales como unidades fisonómico-estructurales-fenológicas, considerando como factores mesológicos las formaciones superficiales (suelos, sedimentos y paleosuelos, principalmente), la unidad hidrogeomorfológica y las condiciones bioclimáticas en las que están insertadas (Cámara, Salva, et al. 2014).

La estructura de la formación se expresa en un gráfico que denominamos Diagrama Ecodinámico de Riqueza-Estructura-Cobertura (DEREC), cuya elaboración se realiza sobre un diagrama binario con un eje vertical y otro horizontal (tabla 1).

Tabla 1. Estructura vertical en el diagrama DEREC

\begin{tabular}{|l|r|}
\hline Estratos & Realidad \\
\hline Arbóreo 4 muy alto (A4) & $>20 \mathrm{~m}$ \\
Arbóreo 3 alto (A3) & $10-20 \mathrm{~m}$ \\
Arbóreo 2 (A2) & $5-10 \mathrm{~m}$ \\
Arbóreo 1 (bajo) (A1) & $3,5-5 \mathrm{~m}$ \\
Arbustivo alto (ab_a) & $1,5-3,5 \mathrm{~m}$ \\
Arbustivo (ab) & $0,6-1,5 \mathrm{~m}$ \\
Subarbustivo (s_ab) & $0,3-0,6 \mathrm{~m}$ \\
Herbáceo (h) & $<0,3 \mathrm{~m}$ \\
\hline
\end{tabular}

La anchura de la barra horizontal en cada estrato se construye con el número de especies (por ejemplo: 10 especies se distribuyen 5 a cada lado del eje; 11 especies, 5,5 a cada lado del mismo). En la superficie de la barra resultante, se incorporan diferentes tramas según la fracción de cabida del estrato (0-10\%; 10-25\%; 25-40\%; 40-60\%; > 60\%).

De forma lateral a las barras, que expresan la riqueza de cada estrato, se incorpora una simbología dinámica de la formación vegetal y de transformación antrópica. Con 
ello se permite obtener por estrato una evolución de la tendencia a disminuir/aumentar en cobertura o en especies. Para establecer la dinámica de las formaciones vegetales se toma como modelo el DEREC que se identifique como situación ecológicamente más madura de la formación vegetal muestreada, y se analizan comparativamente los otros DEREC obtenidos de la misma formación vegetal en otras parcelas.

Como referencia del estado de la vegetación se han utilizado las ortofotos aéreas de 1947 de la Fototeca de Instituto Geográfico Nacional, y fotogramas en Color de 0.5 m del año 2009 (PNOA) de la Junta de Andalucía. Para la distribución actual de la vegetación se ha realizado una clasificación de la imagen Komposat 2 del 06/09/2009 con el método supervisado de máxima verosimilitud, a partir de training sites (muestra de referencia de campo) tomadas por GPS en el área de estudio.

Se localizan y se georreferencian en las salidas de campo los puntos de inicio y final de los transectos, recogiendo las coordenadas con el GPS Global positioning System (Garmin modelo Oregon 550t), (tabla 2).

Para el análisis de la diversidad alfa se han utilizado:

- Métodos paramétricos (Magurran, 1988) como son la curva rango-abundancia, los modelos para los patrones de distribución de especies, y la curva $\mathrm{K}$ dominancia, calculados con los paquetes Vegan (Community Ecology Package) (Dixon, 2003: http://cran.r-project.org/web/packages/vegan/index.html) y Biodiversity R (Kindt, 2014: http://cran.r-project.org/web/packages/BiodiversityR/index.html) del software estadístico R.

- Los índices no paramétricos de diversidad alfa (Moreno, 2001) de Shannon, Simpson, Equitabilidad J y Berger-Parker.

\section{Resultados}

Se ha estudiado la caracterización en atributos (abundancia, dominancia, riqueza) y los índices de diversidad para establecer los cambios de diversidad biológica alfa de las formaciones leñosas de la vegetación. Para ello se ha considerado la eliminación de la vegetación natural en el Médano del Asperillo desde mediados del siglo XX, y su sustitución por un matorral de monte blanco y de Corema album tras 70 años de dinámica.

Se han identificado dos sectores en el médano del Asperillo. El primer sector ubicado en el lugar de Las Atarazanas, está ocupado por un sabinar que permanece, al menos, desde el año 1947, en la cubeta de una duna parabólica fitoestabilizada, con una formación cerrada de pino piñonero (Pinus pinea L.). El segundo sector se localiza en la proximidad de la caminería de uso público de Cuesta Maneli, con un matorral de colonización de camarina (Corema álbum (L.) D. Don) en el blowout de la duna, 
Tabla 2. Datos GPS de principio y fin de cada parcela de las dos muestras: SATv refieren al sabinar de Las Atarazanas y CMv al matorral de Cuesta Maneli

\begin{tabular}{|l|c|c|c|c|}
\hline Parcela & $X_{i}$ & $Y_{i}$ & $X_{f}$ & $Y_{f}$ \\
\hline SATv_1 & 705082 & 4105884 & 705070 & 4105831 \\
SATv_2 & 705070 & 4105831 & 705053 & 4105788 \\
SATv_3 & 705061 & 4105891 & 705039 & 4105845 \\
SATv_4 & 705039 & 4105845 & 705011 & 4105802 \\
SATv_5 & 705011 & 4105802 & 704976 & 4105755 \\
SATv_6 & 704976 & 4105755 & 704961 & 4105705 \\
SATv_7 & 704982 & 4105881 & 704944 & 4105846 \\
SATv_8 & 704944 & 4105846 & 704932 & 4105798 \\
SATv_9 & 704932 & 4105798 & 704933 & 4105750 \\
SATv_10 & 704933 & 4105750 & 704930 & 4105701 \\
CMv_1 & 705358 & 4105633 & 705361 & 4105586 \\
CMv_2 & 705361 & 4105586 & 705375 & 4105544 \\
CMv_3 & 705375 & 4105544 & 705392 & 4105497 \\
CMv_4 & 705392 & 4105497 & 705407 & 4105448 \\
CMv_6 & 705310 & 4105568 & 705313 & 4105523 \\
CMv_7 & 705313 & 4105523 & 705332 & 4105469 \\
CMv_8 & 705332 & 4105469 & 705357 & 4105426 \\
CMv_9 & 705357 & 4105426 & 705376 & 4105389 \\
CMv_10 & 705417 & 4105499 & 705416 & 4105544 \\
CMv_11 & 705416 & 4105544 & 705390 & 4105592 \\
\hline
\end{tabular}

y en las partes más altas un matorral de monte blanco con (Halimium calycinum (L.) K.Koch), y Cistus salvifolius L., sobre arenas móviles. Para poder comparar estos sectores y establecer la degradación del sabinar en el médano del Asperillo se ha realizado 20 transectos de 50 × 2 m.: 10 en Cuesta Maneli y 10 en Las Atarazanas.

La distribución actual de las comunidades vegetales en el Médano del Asperillo está constituido, tal como refleja la imagen de satélite interpretada (figura 3), por:

- Una comunidad arbórea semicerrada de Juniperus phoenicea subsp. turbinata (Guss.) Nyman acompañada de un matorral cerrado de monte blanco con Rosmarinus officinalis L. y Cistus salviifolius L. que ocupa las cubetas de las dunas parabólicas desarrolladas en el médano del Asperillo. Se distribuye también en topografías bajas junto a la carretera, situado en la base del frente dunar que constituye el Médano del Asperillo. 


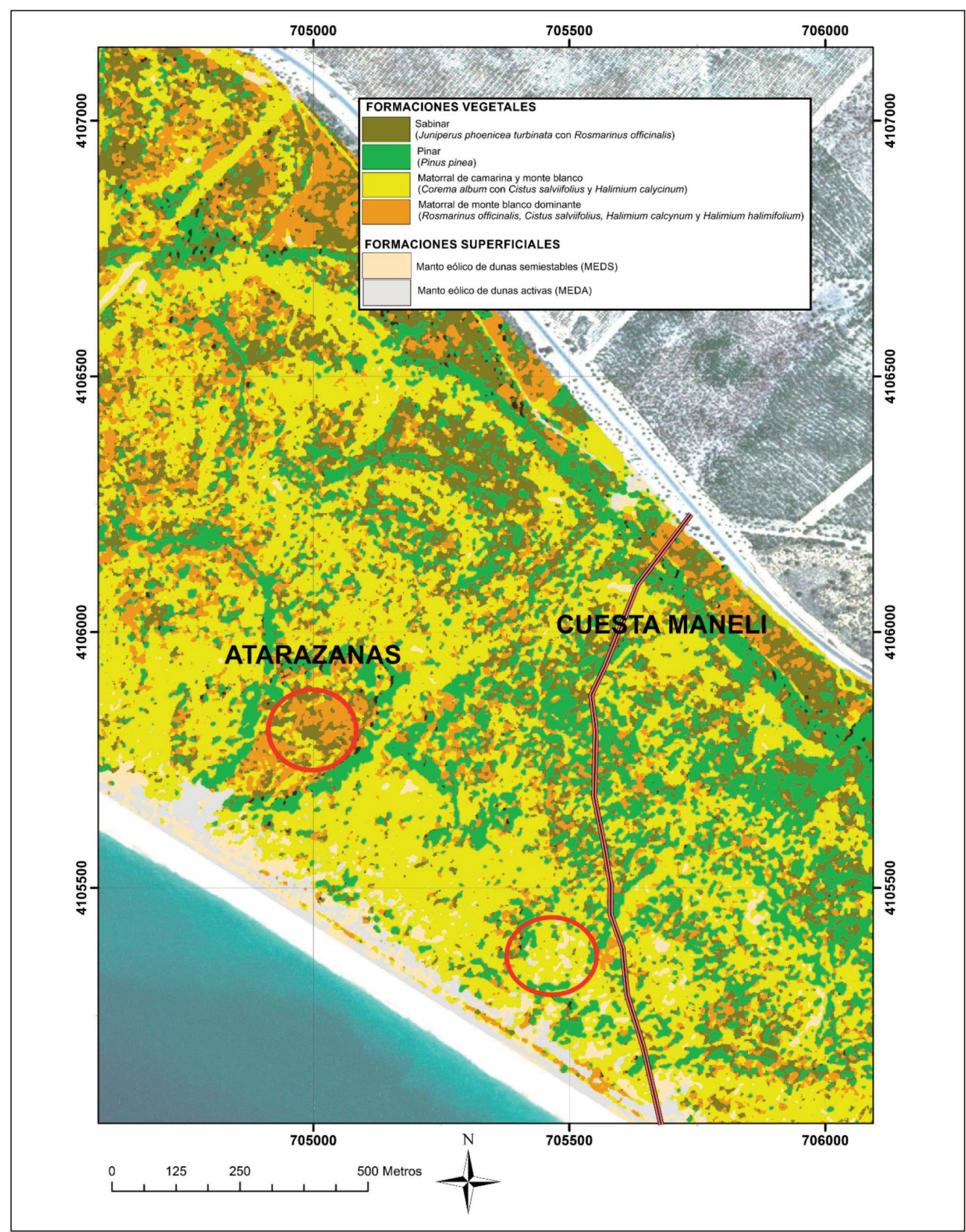

Figura 3. Imagen clasificada del entorno del área de estudio en Las Atarazanas-Cuesta Maneli identificados con círculos rojos. Elaboración propia a partir del análisis y clasificación de la imagen Komposat 2 del 06/09/2009 con el método supervisado de máxima verosimilitud. 
- Una comunidad monoespecífica sin matorral de Pinus Pinea L. sobre las crestas de las dunas parabólicas, y un desarrollo importante en las proximidades de la caminería de uso público de Cuesta Maneli.

- En la superficie que estaba desprovista de vegetación en el año 1947 aparece un comunidad constituida por un matorral de sucesión con Cistus salvifolius L. y Halimium calycinum (L.) K. Koch como dominantes (ocupando los blowout Corema album (L.) D. Don in Sweet como especie dominante).

\subsection{Matorral abierto sobre dunas no estabilizadas}

En Cuesta Maneli, Se han realizado 10 transectos denominados CMv. En esta muestra identificamos una riqueza de 14 especies en 931 individuos censados. La especie con mayor abundancia es Corema album (L.) D. Don in Sweet (313 individuos), acompañada de Halimium calycinum (L.) K. Koch (Cistaceae) (188 individuos) y Cistus salvifolius L. (134 individuos). La mayor dominancia la tiene también Corema album (L.) D. Don in Sweet con una fracción de cabida del 30\%, seguido de Pinus pinea L, con un 7\%. El IVI nos relaciona la presencia, con la abundancia y la dominancia en la muestra (tabla 3), destacando de nuevo Corema album (L.) D. Don in Sweet con Cistus salvifolius L. y Halimium calycinum (L.) K. Koch.

Tabla 3. Valores de IVI en porcentaje de la muestra de Cuesta Maneli (10 parcelas)

\begin{tabular}{|l|c|}
\hline Especies & IVI \\
\hline Corema álbum (L.) D. Don in Sweet & 36.75 \\
Cistus salviifolius L. & 11.56 \\
Halimium calycinum (L.) K. Koch & 11.38 \\
Pinus pinea L. & 8.46 \\
Stauracanthus genistoides (Brot.) Samp. & 7.04 \\
Cytisus grandiflorus (Brot.) DC. & 5.45 \\
Halimium halimifolium (L.) Willk. & 5.26 \\
Juniperus phoenicea subsp. turbinata (Guss.) Nyman & 3.39 \\
Genista anglica L. & 3.37 \\
Rosmarinus officinalis L. & 2.75 \\
Osyris lanceolata Hochst. \& Steud. & 2.32 \\
Cistus libanotis L. & 0.88 \\
Helichrysum picardii Boiss. \& Reuter in Boiss. & 0.81 \\
Lavandula stoechas L. & 0.57 \\
\hline
\end{tabular}

Fuente: Elaboración propia. 
La estructura de la pirámide muestra una mayor riqueza en el estrato arbustivo y subarbustivo con 12 especies, mientras que el resto de los estratos son muy abiertos, con algunos arbustos altos muy localizados por encima de $1.5 \mathrm{~m}$., y de forma testimonial algunos individuos de Pinus pinea L. y Juniperus phoenicea subsp. turbinata (Guss.) Nyman, de más de $3 \mathrm{~m}$. (figura 4).

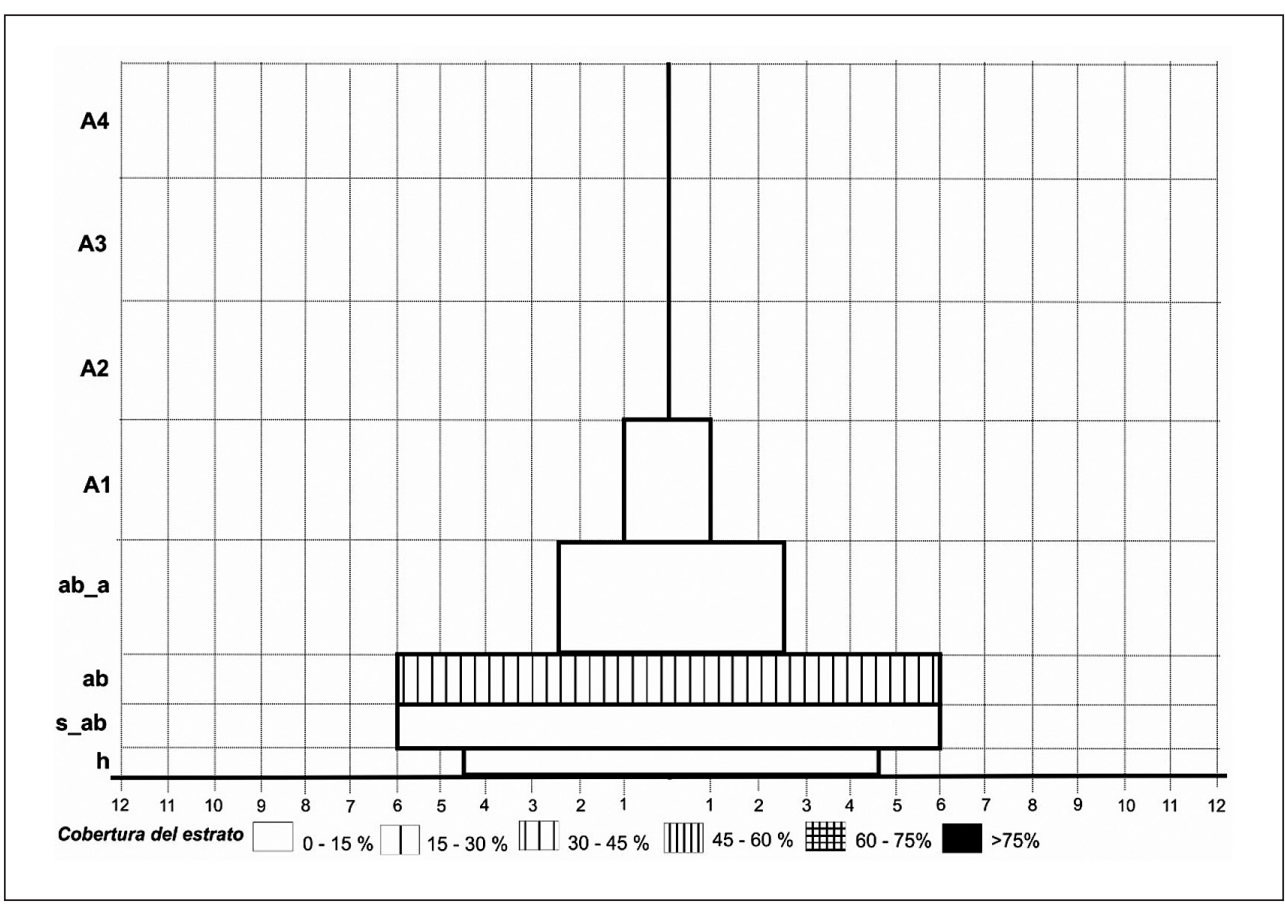

Figura 4. DEREC de síntesis de la muestra de matorral en Cuesta Maneli.

Fuente: Elaboración propia.

Se trata de un matorral monoespecífico de camarina (Corema album (L.) D. Don in Sweet) que ocupa los blowout acompañado monte blanco con Cistus salvifolius L. y Halimium calycinum (L.) K. Koch con una cobertura dominante en el estrato arbustivo entre 0,60 y $1,50 \mathrm{~m}$. cuya fracción de cabida es de 43\%, quedando más de la mitad de la superficie de arenas sin cubrir.

Los valores de diversidad alfa no paramétricos para esta formación dan un índice de Shannon de 1.9 nats, un valor de Simpson de 0.8, Berger-Parker 0.34, y un índice de equitabilidad-J de 0.73 . Estos índices muestran una baja a media diversidad, con alta dominancia y una equitabilidad media a alta que marca la ausencia de especies raras y un reparto homogéneo de individuos entre las especies que no son dominantes. 


\subsection{Sabinar del sector La Atarazana}

En el área denominada La Atarazana nos encontramos con un sistema vegetativo que debía ser el propio de la zona del Asperillo sobre un sistema de dunas estabilizadas.

La estructura arbórea de pinar se sitúa en la parte alta de las crestas de la duna que cierra al este y por los lados la cubeta dunar, espacio protegido del viento, en la que se sitúa un bosque cerrado de sabinos.

En el área de La Atarazana se han realizado una muestra de $1.000 \mathrm{~m}^{2}$, con 10 parcelas de $50 \times 2 \mathrm{~m}$. En ella identificamos una riqueza de 9 especies con 1.284 individuos censados. Las que presentaban mayor abundancia eran Rosmarinus officinalis L. (551 individuos), Cistus salviifolius L. (381 individuos) y Juniperus phoenicea subsp. turbinata (Guss.) Nyman, (144 individuos). La mayor dominancia es de Juniperus phoenicea subsp. turbinata (Guss.) Nyman, con una fracción de cabida del 28\%, seguido de Rosmarinus officinalis L. con un 6\% y Corema album (L.) D. Don in Sweet con el 4,5\%. En el IVI de la muestra (tabla 4) destaca de nuevo Juniperus phoenicea subsp. turbinata (Guss.) Nyman con Rosmarinus officinalis L. y Cistus salviifolius L.

Tabla 4. Valores de IVI en porcentaje de la muestra de Las Atarazanas (10 parcelas)

\begin{tabular}{|l|c|}
\hline Especies & IVI \\
\hline Juniperus phoenicea subsp. turbinata (Guss.) Nyman & 30.34 \\
Rosmarinus officinalis L. & 24.58 \\
Cistus salviifolius L. & 16.75 \\
Corema álbum (L.) D. Don in Sweet & 11.89 \\
Osyris lanceolata Hochst. \& Steud. & 5.59 \\
Halimium calycinum (L.) K. Koch & 5.17 \\
Halimium halimifolium (L.) Willk. & 3.43 \\
Pinus pinea L. & 1.65 \\
Daphne gnidium L. & 0.60 \\
\hline
\end{tabular}

Fuente: Elaboración propia.

La estructura de la pirámide del sabinar de la Atarazana muestra una mayor riqueza en el estrato arbustivo, con 12 especies, mientras que el resto de los estratos son muy abiertos, con algunos arbustos altos muy localizados por encima de $1.5 \mathrm{~m}$., y de forma testimonial algunos individuos de pinos de más de $3 \mathrm{~m}$. (figura 5). 


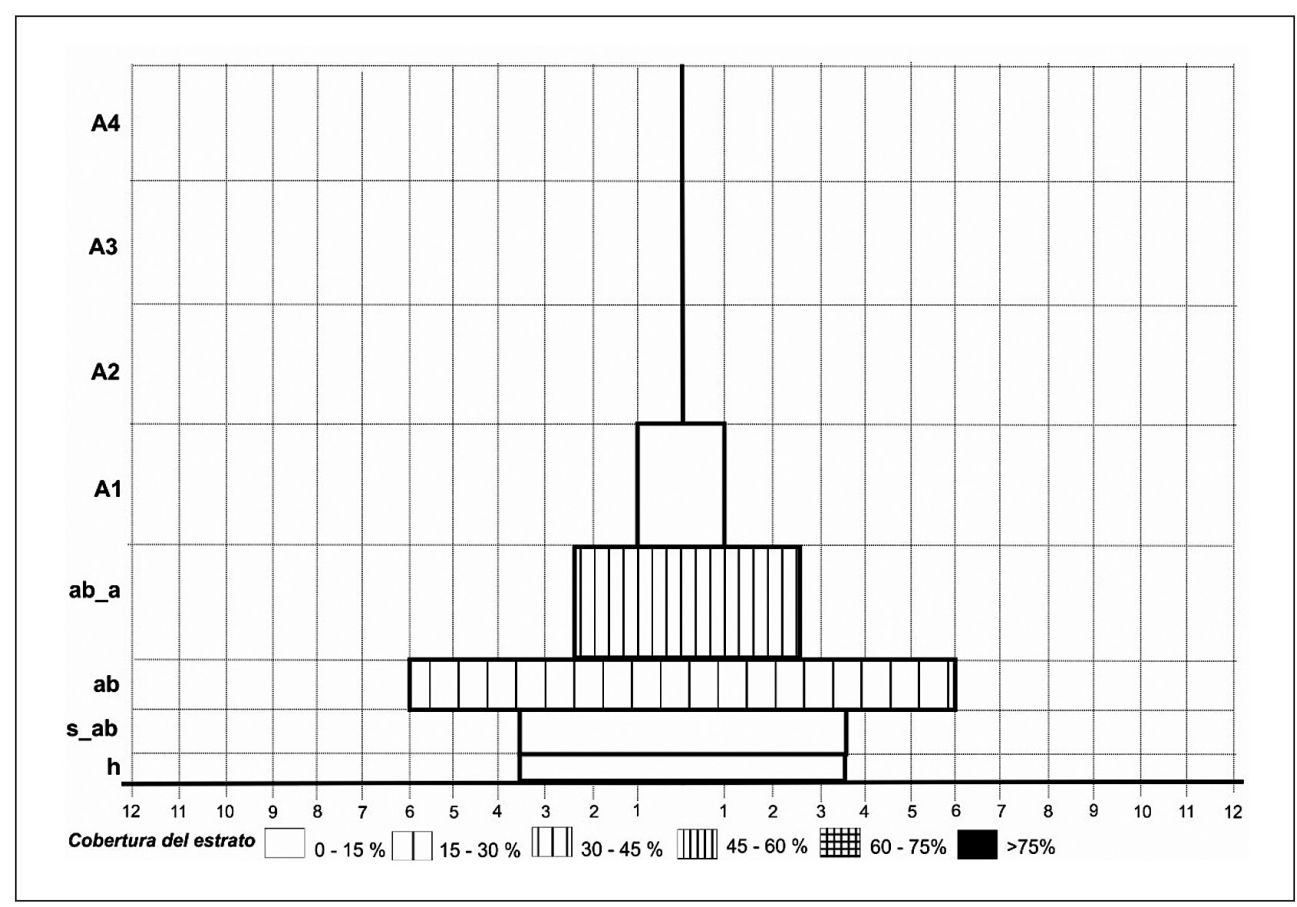

Figura 5. DEREC de síntesis de la muestra de matorral en Atarazanas.

Fuente: Elaboración propia.

Se trata de un bosque semicerrado monoespecífico de sabinar Juniperus phoenicea subsp. turbinata (Guss.) Nyman de altura media de $3 \mathrm{~m}$. acompañado de un monte blanco constituido con Rosmarinus officinalis L. y Cistus salviifolius L.

Los valores de diversidad alfa no paramétricos para esta formación dan un índice de Shannon de 1,45 nats y un valor de Simpson de 0,7, un valor de Berger-Parker de 0,43 y un índice de Equitabilidad-J de 0,66. Esto marca una baja diversidad alfa con alta dominancia de unas especies sobre el resto pero con una equitabilidad media Es decir, que si bien hay algunas especies dominantes, el resto de los individuos está repartido homogéneamente entre el resto de las especies, o lo que es lo mismo, no hay especies raras.

\subsection{Análisis comparativo de la biodiversidad alfa de las dos comunidades vegetales}

La curva especies-abundancia (figura 6) marca una mayor diversidad para el matorral de Cuesta Maneli (mCMA) que para el sabinar de Las Atarazana (sATA), ya que la curva de la primera comunidad está más desplazada hacia la derecha. 


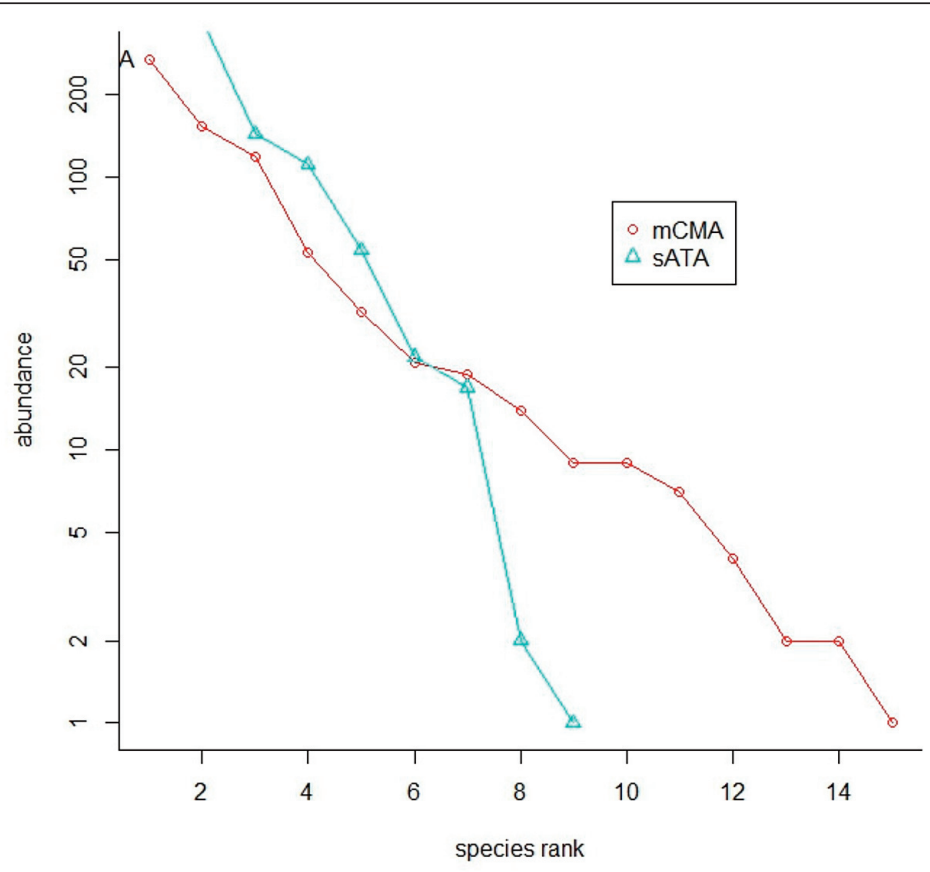

Figura 6. Curva especies-abundancia para el matorral Cuesta Maneli (mCMA) y sabinar de Las Atarazanas (SATA).

Fuente: Elaboración propia a partir del software R.

Los modelos de patrones de distribución de especies (figura 7) muestran la relación existente entre la cantidad de individuos que puede alcanzar una especie en la comunidad y la cantidad de recursos que se apropia. Si una especie concentra la mayor parte de los individuos en unos pocos tipos de recursos, y estos son escasos, será menos abundante que una que muestre mayor amplitud. Así hay distintos modelos sobre cómo se reparten los recursos entre las especies de una comunidad que producen distintos patrones de abundancia relativa (Magurran. 1988). En nuestro caso de estudio los modelos existentes son Preemption para el sabinar de Las Atarazanas y Mandelbrot para el matorral de Cuesta Maneli.

En el modelo preemption o serie geométrica unas pocas especies son dominantes con las restantes prácticamente raras y por ello es la menos equitativa. Por su parte el modelo Mandelbrot, o Serie logarítmica, resulta cuando uno o unos pocos factores dominan la ecología de la comunidad, como en las etapas iniciales de sucesión (Magurran, 1988; Pielou 1975; Krebs, 1989). 


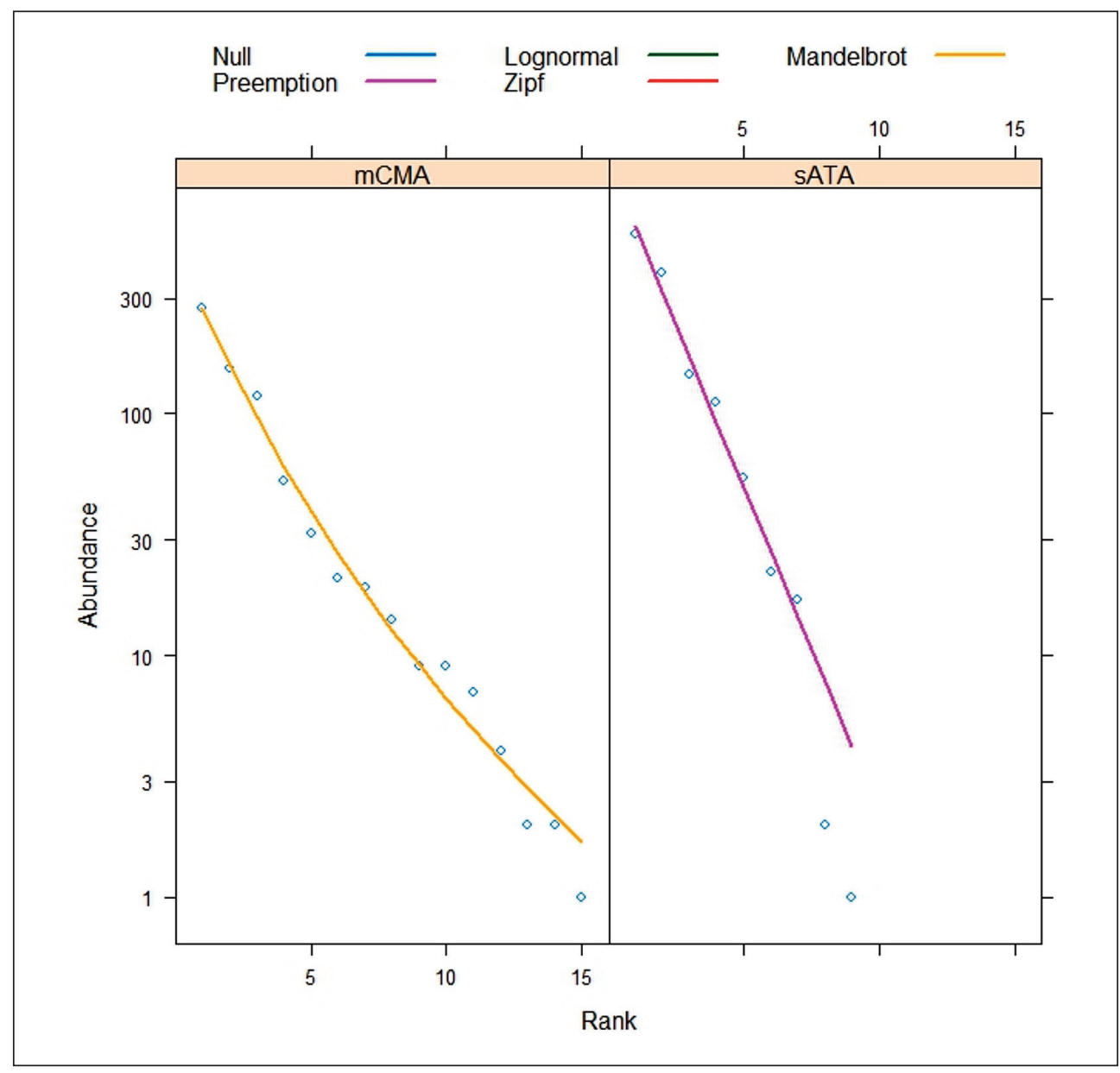

Figura 7. Modelos para los patrones de distribución de especies para el matorral Cuesta Maneli (mCMA) y sabinar de Las Atarazanas (sATA).

Fuente: Elaboración propia a partir del software R.

La curva K-dominancia es una herramienta para la medición de las tendencias de abundancia en las comunidades a través del tiempo (Jennings et al. 2001). La lógica que hay detrás de esta curva es que sólo el subconjunto de especies que pueden tolerar la perturbación va a prosperar y el resto se reducirá o desaparecerá. Por lo tanto, la curva más pronunciada y más elevada muestra la diversidad más baja y el estado del sistema más perturbado (Rice, 2000). En el caso de Las Atarazanas-Cuesta Maneli, (figura 8) muestra una menor diversidad y una mayor perturbación para el sabinar. 


\section{grafico dominancia $\mathbf{k}$}

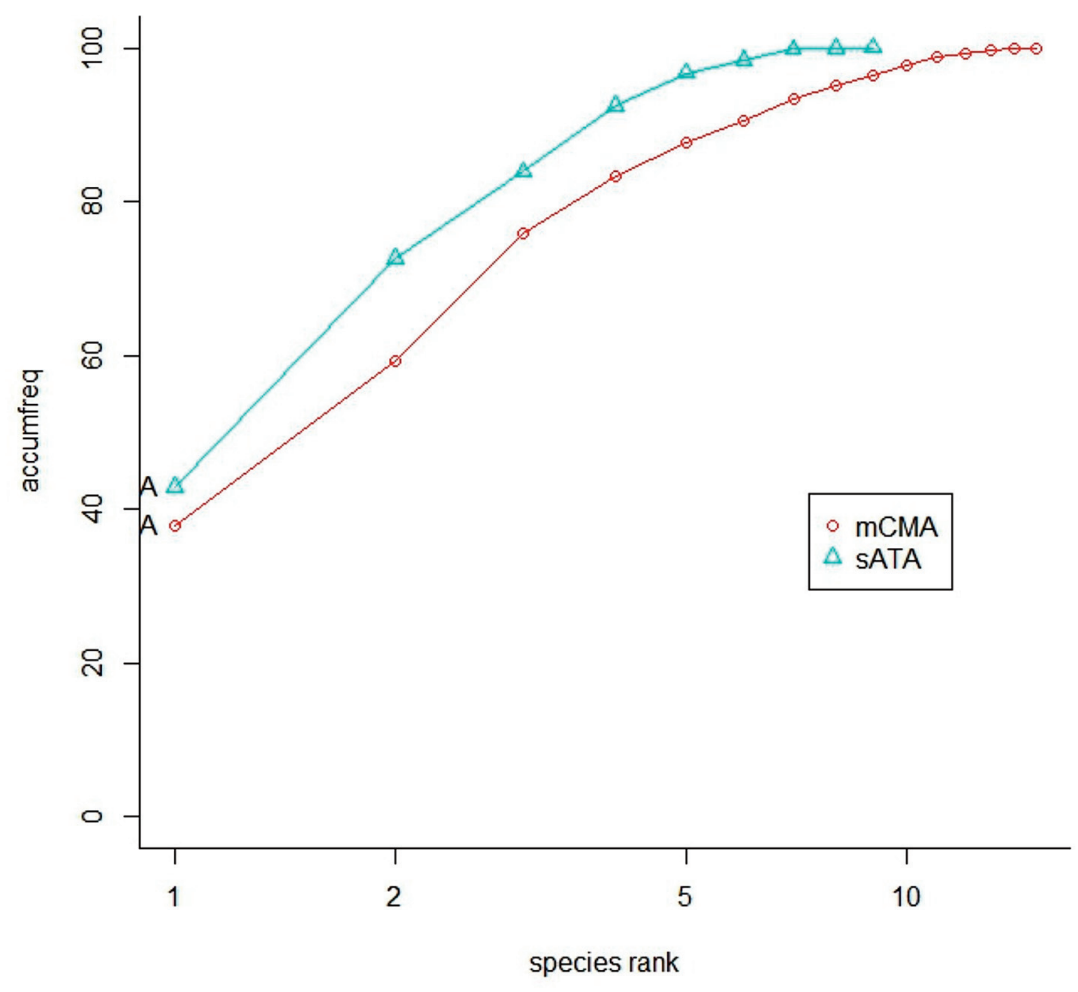

Figura 8. Curvas de K dominancia del matorral de cuesta Maneli (mCMA) y el sabinar de Las Atarazanas (SSATA).

Fuente: Elaboración propia a partir del software R.

\section{Discusión}

Hemos comparado los resultados aquí alcanzados para el sabinar de Las Atarazanas con los del sabinar de El Marqués (Cámara et al., 2014). Para la misma superficie inventariada muestra una mayor riqueza del segundo con respecto al primero (19 especies en El Marqués y 9 en Las Atarazanas), pero con mayor abundancia en Las Atarazanas (1284 frente a 940 individuos). El índice de Shannon es más alto en El Marqués (2,02 nats) que en Las Atarazanas (1,45 nats). Respecto a las especies el IVI marca una alta semejanza en los dos sabinares, con una mayor valor para Juniperus phoenicea subsp. turbinata (Guss.) Nyman, Rosmarinus officinalis L., y Cistus salviifolius L. La di- 
ferencia entre ambos lugares radica en la presencia de Corema album (L.) D. Don in Sweet en Las Atarazanas, que en El Marqués sólo es testimonial. Esta situación muestra, dos sabinares de características similares que han permanecido desde 1947, pero que en caso de Las Atarazanas presenta una menor biodiversidad alfa y una menor riqueza. Ecológicamente el sabinar de las Atarazanas no presenta ningún tipo de evolución edáfica en las cubetas dunares, mientras que en los sabinares de El Marqués y El Ojillo se han descrito la formación de un arenosol lúvico y crómico (Recio et al. 2014).

El estudio realizado con el mismo método en los sabinares de la Reserva Biológica de Doñana (Cámara, Gómez et al., 2014) con 39 parcelas $\left(3.900 \mathrm{~m}^{2}\right.$ de superficie inventariados) identificó 26 especies con 1968 individuos, con un índice de Shannon general de 2,11 nats y una equitabilidad J de 0,65. Estos resultados acercan al sabinar de El Marqués a los valores generales para el conjunto del sabinar de Doñana, pero deja desmarcado al de Las Atarazanas.

Se ha realizado un análisis comparativo de la estructura del matorral de sucesión con el sabinar. Se marca en el diagrama DEREC de síntesis (figura 9) una tendencia a aumentar en cobertura el estrato arbustivo alto con la misma riqueza (5 especies), aunque aumentando la dominancia y abundancia de Juniperus phoenicea subsp. turbinata (Guss.) Nyman, y por lo tanto, por la mayor cobertura del estrato superior, tiende a disminuir la del estrato arbustivo, al igual que la riqueza de éste y de los estratos que se encuentra por debajo de él.

Las especies presentes en las dos comunidades son Cistus salvifolius L., Corema album (L.) D. Don in Sweet, Halimium calycinum (L.) K. Koch, y con menor peso Halimium halimifolium (L.) Wilk. y Osyris lanceolata Hochst \& Steud., que constituyen la conexión de especies de matorral entre las dos comunidades vegetales estudiadas. La importante presencia en abundancia y dominancia en el sabinar de Juniperus phoenicea subsp. turbinata (Guss.) Nyman con Rosmarinus officinalis L. marca la principal diferencia entre ambas comunidades a nivel de presencia, abundancia y dominancia de especies. Esta circunstancia es corroborada por los índices de Shannon ya indicados para cada comunidad.

Por su parte las curvas ABC (Abundance/Biomass Comparison) utilizan las curvas de K-dominancia para determinar los niveles de perturbación en las comunidades. Al no disponer de datos de biomasa hemos utilizado los datos de dominancia con la cobertura, que está directamente relacionada con la biomasa (CATIE, 1998 pp. 44, Isaza y Betancur, 2009). La curva de biomasa estará por encima de la curva de abundancia en ambientes no perturbados, mientras que sucede lo inverso en ambientes perturbados (Warwick et al. 1986). Por el contrario, en ambientes donde la perturbación es moderada, ambas curvas tienden a coincidir de manera aproximada o sobreponerse una sobre otra. Según Warwick: 


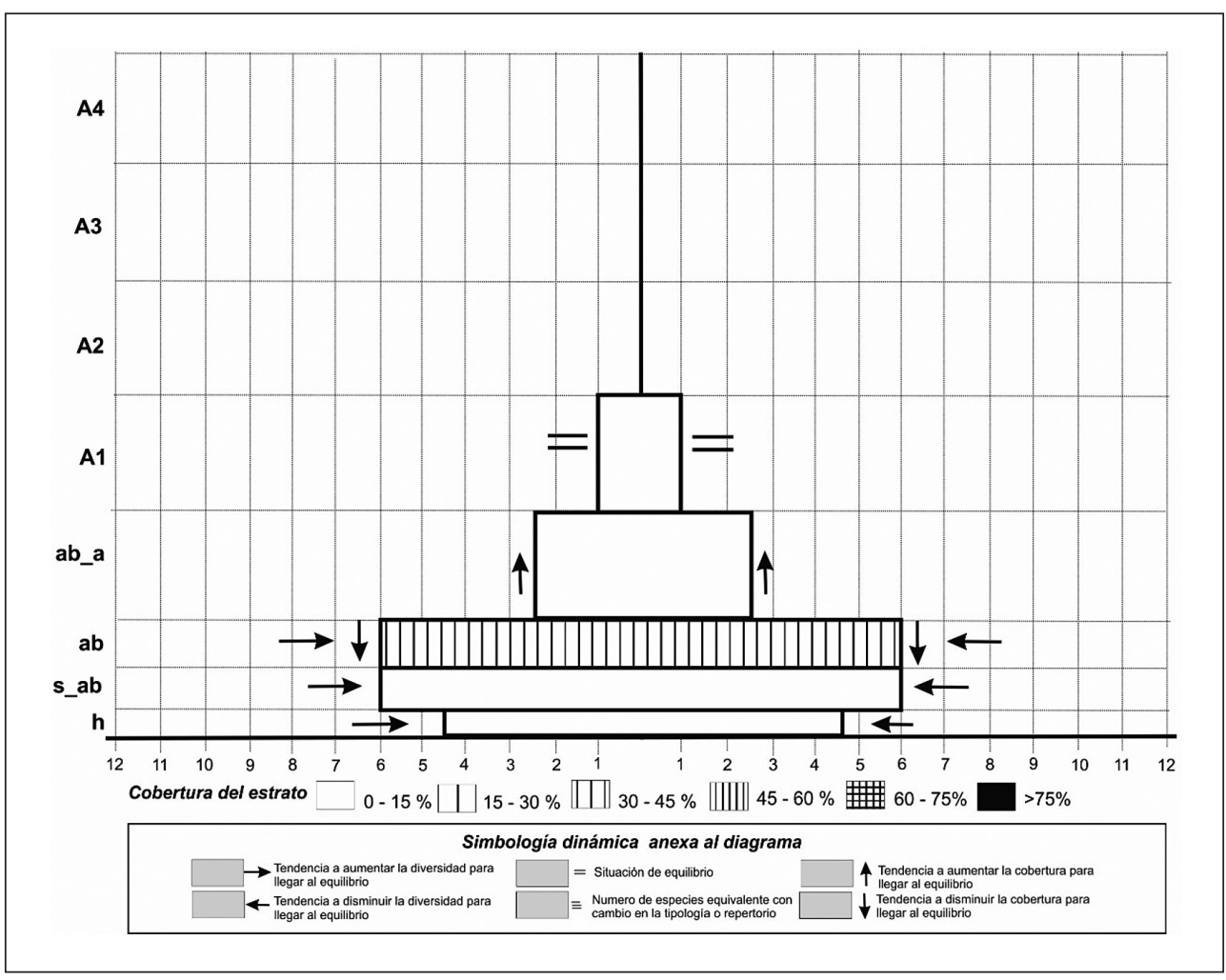

Figura 9. DEREC con expresión dinámica de riqueza y cobertura hacia el DEREC del sabinar. Fuente: Elaboración propia.

- Cuando la comunidad se aproxima al equilibrio, la biomasa tiende a estar dominada por unas pocas especies con individuos grandes y poco numerosos, y la dominancia numérica sigue estando mantenida por especies pequeñas. Esto da lugar a una mayor equitatividad de la abundancia entre las especies que en la biomasa.

- Bajo condiciones de estrés moderado, las especies dominantes grandes son eliminadas, resultando una menor diferencia entre los índices de equitatividad en términos de biomasa y abundancia.

- Bajo condiciones severas de perturbación ambiental, las pocas especies grandes que quedan contribuirán proporcionalmente más a la biomasa total de la comunidad con relación a su abundancia que como lo hacen las especies pequeñas dominantes.

- Como efecto neto, cuanto mayor es el estrés, la biomasa estará más homogéneamente distribuida entre las especies que el número de individuos. 
A partir de sus premisas, Warwick crea un método de bioindicación ambiental fundamentado en graficar las curvas de K-dominancia basadas una en abundancia en número y la otra en biomasa (en nuestro caso dominancia): cuanto más por encima esté en la gráfica la curva de abundancia de la de dominancia, más desfavorables son las condiciones ambientales. Como se puede observar en la figura 10 el matorral de Cuesta Maneli se encuentra en condiciones ambientales más favorables que el sabinar de las Atarazanas, en el que la curva de abundancia llega a estar por encima de la de dominancia, lo que muestra, además, una mayor perturbación en el sabinar que en matorral de sucesión. El matorral de sucesión en Cuesta Maneli muestra sin embargo una situación de gran equilibrio, con la curva de dominancia claramente por encima de lla de abundancia.

\section{Conclusiones}

El estudio comparativo de los atributos e índices de biodiversidad del matorral de Cuesta Maneli y el Sabinar de Las Atarazanas pone de manifiesto que, si bien el sabinar se ha mantenido desde el año 1947, tal como reflejan los fotogramas aéreos, presenta unos índices de diversidad y de estado ambiental por debajo del matorral de sucesión distribuido en el entorno de Cuesta Maneli, así como de otros sabinares (sabinar de El Marqués en la Reserva Biológica de Doñana).

El repertorio de especies de ambas comunidades marca una relación entre ellas, de tal manera que la única diferencia, entre el matorral de sucesión y el sabinar, es la mayor abundancia y dominancia de Juniperus phoenicea subsp. turbinata (Guss.) Nyman y Rosmarinus officinalis L. y la desaparición en el sabinar de Stauracanthus genistoides (Brot.) Samp. Cytisus grandiflorus (Brot.) DC. y Halimium halimifolium (L.) Willk.

La hipótesis planteada inicialmente de un estado de preservación más naturalizado con el sabinar por su permanencia, no se cumple. La alta abundancia y dominancia de Rosmarinus officinalis L. y los resultados de los índices de biodiversidad y de preservación ambiental (k-abundancia/dominancia) indican que el sabinar, a pesar de su permanencia, es una comunidad con una fuerte perturbación, seguramente por uso humano. El ritmo de ocupación de la superficie de arenas inestables en 70 años ha permitido la formación de dunas fitoestables, pasando de una situación de morfogénesis activa a otra de morfogénesis muy limitada por la vegetación, de tal manera que el ritmo de cambio en el balance geomorfológico estabilidad-inestabilidad ha sido más rápido que el de sucesión de la vegetación.

Estos resultados invitan a seguir profundizando en el conocimiento de la dinámica de las comunidades vegetales y los cambios que se producen en su estructura, composición florística y biodiversidad, ya que el estudio aquí realizado pone de manifiesto 


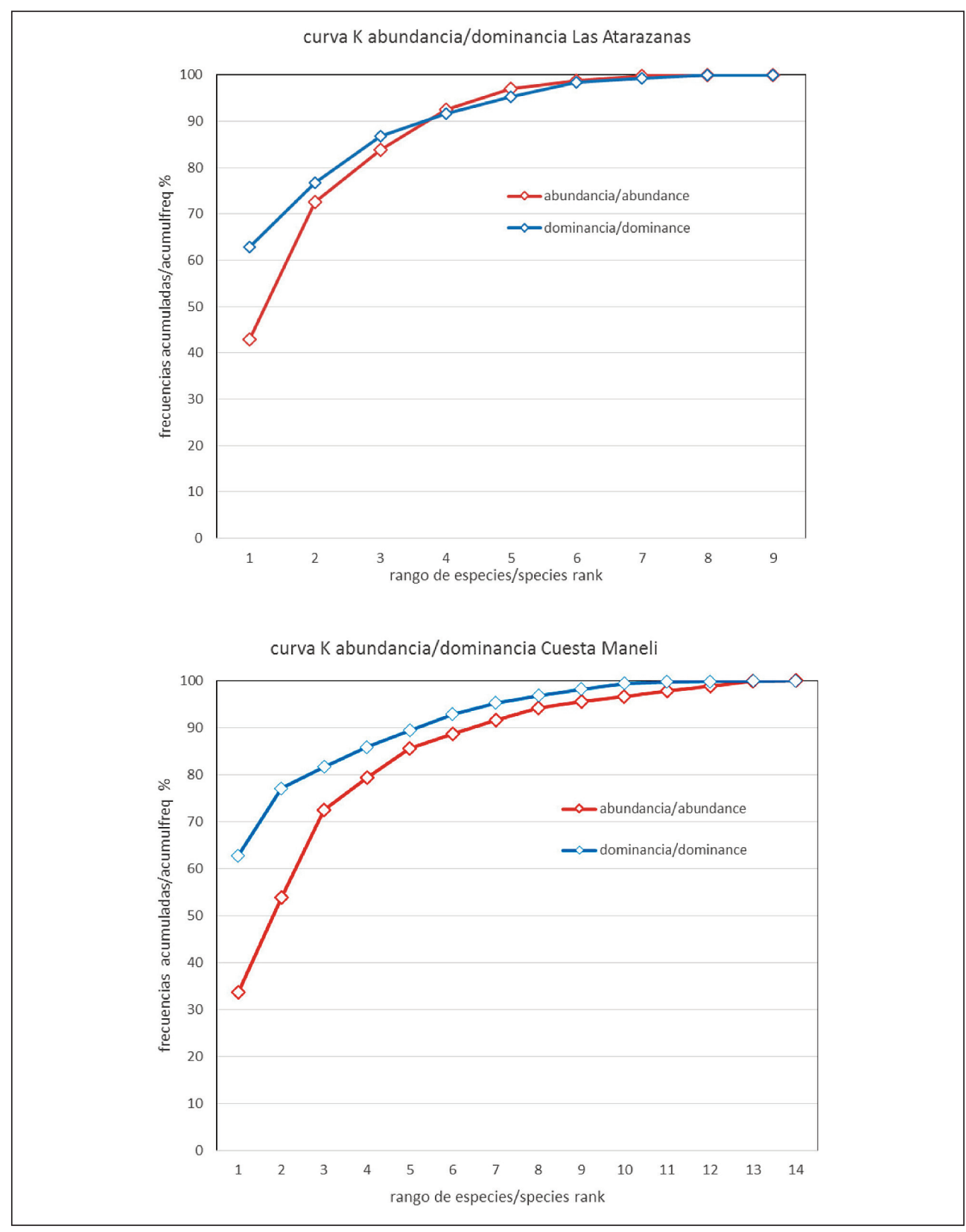

Figura 10. Curvas K-abundancia/dominancia para el sabinar de Las Atarazanas (arriba) y el matorral de Cuesta Maneli (abajo).

Fuente: Elaboración propia. 
el desconocimiento relativo a estas sucesiones en medios litorales sobre dunas de forma particular, y en otros ámbitos en general. El análisis aquí presentado sirve también para establecer el grado de conservación/degradación de una comunidad vegetal, más allá de lo que pueda transmitir su permanencia temporal en un lugar.

\section{Agradecimientos}

Al proyecto JUNITUR CSO2011-24425 “Biogeografía de los sabinares de Juniperus turbinata Guss en la Reserva de la Biosfera de El Hierro y en el Parque Nacional de Doñana", bajo cuya financiación se llevó acabo esta investigación.

\section{Materiales}

- Red de Información Ambiental de Andalucía. Consejería de Medio Ambiente. Junta de Andalucía. Sevilla. 2000.

- Vuelo Ortofotográfico de Andalucía. Color de 0.5 m (PNOA). Hoja 1021. Instituto Cartográfico de Andalucía (ICA), Sevilla. 2010.

- Vuelo de 1947. Hoja 1021. Fototeca de Instituto Geográfico Nacional.

- Imagen de satélite de alta definición Komposat 2 de fecha 06/09/2009, suministrada por Aurensis.

\section{Bibliografía}

Bejarano, R., 1994. Los paisajes vegetales de la costa onubense y su significación. Huelva en su Historia, 5, 157-186.

Bejarano, R., 1997. El análisis de la vegetación como criterio de interpretación del paisaje (Andalucía atlántica). Estudios Geográficos, Vol. 58 Núm. 226. 5-32.

Bejarano, R., 2004. Tipología de los sabinares costeros según su estructura vertical y el grado de cobertura por especies", En: J. M. Panareda et al. (eds.): Estudios en Biogeografía. Libro Homenaje a José Manuel Rubio Recio y Jesús García. Terrasa, Aster, pp. 59-74.

Bejarano, R., Cámara, R., Borja, C., Díaz del Olmo, F., Recio, J.M. y Borja, F., 2010. Caracterización de los bosques de sabina (Juniperas turbinata Guss.) del entorno de la laguna Charco del Toro (Parque Nacional de Doñana, Huelva): aplicación de una nueva metodología para el estudio de la vegetación. En: P. Giménez, J. A. Marco, E. Matarredona, A. Padilla y A. Sánchez (eds.): Biogeografía: una ciencia para la conservación del medio. VI Congreso Español de Biogeografía. Alicante. Universidad de Alicante y Asociación de Geógrafos Españoles, 25-34.

Borja, F., Díaz del Olmo, F., 1994a. El Acantilado de El Asperillo: Cuaternario Reciente y Fases Históricas en el Litoral de Huelva. Geogaceta. Vol. 15. 94-99. 
Borja, F. y Díaz del Olmo, F., 1994b. Geomorfología del manto eólico litoral de El Abalario (Huelva). En: Arnáez-Vadillo J., García Ruiz J. M. y Gómez Villar A. (eds.) Geomorfología en España. Logroño, SEG. 327-338.

Cámara, R., Gómez, C., Díaz del Olmo, F., Borja, C., 2014. Cartografía de las formaciones vegetales y unidades ambientales de los mantos eólicos del Parque Nacional Doñana y Reserva Biológica de Doñana. En: Cámara, R., Rodríguez, B., Muriel, J.L. (eds) Biogeografía de Sistemas Litorales. Dinámica y Conservación. Sevilla. Universidad de Sevilla. 203-207.

Cámara, R., Díaz del Olmo, F., 2013. Muestreo en transecto de formaciones vegetales de fanerófitos y Caméfitos (I): Fundamentos metodológicos. Estudios geográficos. Vol. 74. Núm. 274. p. 67-88.

Cámara, R., Díaz del Olmo, F., Borja, C., 2013. Muestreo en transecto de formaciones vegetales de fanerófitos y caméfitos (MIFC) (II): estudio de los sabinares de la Reserva Biológica de Doñana (RBD) (España). Estudios geográficos. Vol. 74. Núm. 274. 89-114.

Cámara, R., Díaz del Olmo, F., Borja, C., Recio, J.M., Salva, M., 2014. Caracterización geobotánica del sabinar de El Marqués. Reserva Biológica de Doñana: factores geoedáficos y termohigrométricos. En: Cámara, R., Rodríguez, B., Muriel, J.L. (eds) Biogeografía de Sistemas Litorales. Dinámica y Conservación. Sevilla. Universidad de Sevilla. 65-72.

Cámara, R., Salva, M., Borja, C., Salvador, F., 2014. Análisis comparado del balance hídrico del suelo y bioclimático de la estación del Palacio de Doñana con los datos termohigrométricos del Sabinar del Marqués (Parque Nacional de Doñana) En: Fernández S. y Rodrigo F.S. Cambio Climático y Cambio Global. Almería. Asociación Española de Climatología. 779-788.

CATIE, 1998. Avances Técnicos, Tomo IX. Enero-Diciembre. San José.

De Las Heras, M.A., 2009. Estudio de flora y vegetación del oeste del entorno de Doñana. Memoria de Tesis Doctoral. Universidad de Huelva.

Díaz Delgado, R. Afán I., Silva, R., 2014. Patrones espaciales de daño en copa en el sabinar de la Reserva Biológica de Doñana a consecuencia de un evento extremo de sequía. En: Cámara, R., Rodríguez, B., Muriel, J.L. (eds) Biogeografía de Sistemas Litorales. Dinámica y Conservación. Sevilla. Universidad de Sevilla. 189-192.

Dixon, P., 2003. VEGAN, a package of R functions for community ecology. Journal of Vegetation Science, Volume 14, Issue 6, pages 927-930.

García Novo, F., Martin, A., Toja, J., 2007. La frontera de Doñana. Universidad de Sevilla-Vicerrectorado de Investigación. Colección Divulgación Científica, oํ 10. Sevilla.

Isaza, C. Betancur, J., 2009. Relación entre biomasa y algunas características morfológicas de bromelias fitotelmatas de un bosque alto andino colombiano. Caldasia Vol. 31, núm. 1. 1-7.

Jennings S., Pinnegar J.K., Polunin, N.V.C., Warr, K.J., 2001. Impacts of trawling disturbance on the trophic structure of bentic invertebrate communities, Marine ecology Progress Series. Vol. 213. $127-142$.

Kindt, R. y Coe, R., 2005. Tree diversity analysis. A manual and software for common statistical methods for ecological and biodiversity studies. Nairobi. World Agroforestry Centre (ICRAF).

Krebs, C.J., 1985. Ecologia: Estudio de distribución y abundancia. México. Segunda edición. Harla México. 
Krebs, C.J., 1989. Ecological methodology. Harper Collins Publ. 654 pp.

López, I., 2009. Vegetación del manto eólico de Doñana. Memoria de Tesis Doctoral. Universidad de Huelva.

Magurran, A.E., 1988. Ecological diversity and its measurement. New Jersey, Princeton University Press.

Montes, C., Borja, F., Bravo, M.A. y Moreira, J.M., 1998. Reconocimiento biofísico de Espacios Naturales Protegidos. Doñana: una aproximación ecosistémica. Sevilla, C.M.A. Junta de Andalucía.

Moreno, C.E., 2001. Métodos para medir la biodiversidad. M\&T-Manuales y Tesis SEA, vol. 1. Zaragoza.

Pielou, E.C., 1975. Ecological diversity. John Wiley \& Sons, Inc., New York.

Warwick, R.M., 1986. A new method for detecting pollution effects on marine macrobenthic communities. Marine Biology, 92. 557-562.

Recio, J.M., Cámara, R., Borja, C., Díaz del Olmo, F., Borja, F., 2014. Edafogénesis sobre el manto eólico litoral del Abalario-Doñana (Huelva, España): el perfil SOJ-2 del Sabinar de El Ojillo. En: Cámara, R., Rodríguez, B., Muriel, J.L. (eds) Biogeografía de Sistemas Litorales. Dinámica y Conservación. Sevilla. Universidad de Sevilla. 73-77.

Rivas-Martínez, S., Costa, M., Castroviejo, S. y Valdés, B., 1980. La vegetación de Doñana (Huelva, España). Lazaroa, 2. 5-190.

Zazo, C., Dabrio, C., Borja, F., Goy, J.L., Lezine, A.M., Lario, J., Polo, M.D. y Hoyos, M., 1999. Pleistocene and Holocene aeolian facies along the Huelva coast (Southern Spain): climatic and neotectonic implications. Geologie in Mijnbouw, 77, 209-224. 
